\title{
Acquisition of methicillin-resistant Staphylococcus aureus after living donor liver transplantation: a retrospective cohort study Masao Hashimoto ${ }^{1}$, Yasuhiko Sugawara*1, Sumihito Tamura1, Junichi Kaneko1, Yuichi Matsui ${ }^{1}$, Junichi Togashi' ${ }^{1}$, Kyoji Moriya ${ }^{2}$, Kazuhiko Koike ${ }^{2}$ and Masatoshi Makuuchi ${ }^{1}$
}

\author{
Address: ${ }^{1}$ Artificial Organ and Transplantation Division, Department of Surgery, University of Tokyo, Tokyo, Japan and ${ }^{2}$ Department of Infectious \\ diseases, University of Tokyo, Tokyo, Japan \\ Email: Masao Hashimoto - hashimom-tky@umin.ac.jp; Yasuhiko Sugawara* - yasusugatky@yahoo.co.jp; Sumihito Tamura - sumitmr- \\ tky@umin.net; Junichi Kaneko - jkaneko-gi@umin.ac.jp; Yuichi Matsui - ymatsui-tky@umin.ac.jp; Junichi Togashi - jtogashi-gi@umin.ac.jp; \\ Kyoji Moriya - moriya-tky@umin.ac.jp; Kazuhiko Koike - kkoike-tky@umin.ac.jp; Masatoshi Makuuchi - makuuchi-tky@umin.ac.jp \\ * Corresponding author
}

Published: II November 2008

BMC Infectious Diseases 2008, 8:155 doi:10.1 I86/147/-2334-8-155

This article is available from: http://www.biomedcentral.com/147/-2334/8/155

(C) 2008 Hashimoto et al; licensee BioMed Central Ltd.

This is an Open Access article distributed under the terms of the Creative Commons Attribution License (http://creativecommons.org/licenses/by/2.0), which permits unrestricted use, distribution, and reproduction in any medium, provided the original work is properly cited.
Received: 18 October 2007

Accepted: II November 2008

\begin{abstract}
Background: The incidence and risk factors of methicillin-resistant Staphylococcus aureus (MRSA) acquisition after living donor liver transplantation (LDLT) are unclear. The aim of the present study was to assess the incidence and to analyze the risk factors for the acquisition of MRSA after LDLT in adults by multivariate analysis.

Methods: We retrospectively reviewed the data from I 58 adult patients that underwent LDLT at the Tokyo University Hospital. The microbiologic and medical records of the patients from admission to 3 months after LDLT were reviewed. Uni- and multivariate analyses were performed to identify the risk factors for postoperative acquisition of MRSA.

Results: Postoperative MRSA acquisition was detected in 35 of 158 patients by median postoperative day 18. Age (>= $60 \mathrm{y})$ and perioperative dialysis and/or apheresis predicted postoperative MRSA acquisition by multivariate analysis. In contrast, postoperative use of fluoroquinolone was negatively associated with acquisition of MRSA.

Conclusion: MRSA arose early after LDLT in adults with a high incidence (35 of 158 patients). Surveillance culture should be checked periodically after LDLT to identify and prevent the transmission of MRSA.
\end{abstract}

\section{Background}

Methicillin-resistant Staphylococcus aureus (MRSA) infection frequently complicates the postoperative course of deceased donor liver transplantation (DDLT) recipients [1-5]. In some centers, 91\% (45 of 49 isolates) of all Staphylococcus aureus infections after DDLT are caused by MRSA [2].
Preoperative MRSA carriage is associated with an increased risk of MRSA infection after DDLT [1,3-5]. Positive MRSA culture in postoperative as well as in preoperative surveillance is important because the finding of MRSA colonization in a patient during hospitalization increases the risk of MRSA infection [6]. In one prospective study [6], the relative risk for developing MRSA infec- 
tion in patients who had MRSA colonization was higher than that in patients who were not colonized with Staphylococcus aureus. In this particular study, 12 of 394 patients had MRSA colonization during hospitalization, and 4 of $12(25 \%)$ later developed MRSA infection.

Few studies have focused on the factors associated with the acquisition of MRSA following liver transplantation. In one prospective study [7], the use of a urinary catheter for a prolonged period, postoperative bleeding at the surgical site, and preoperative use of fluoroquinolones independently increased the risk of MRSA colonization after DDLT. MRSA in cases of living donor liver transplantation (LDLT), in which operations are performed in a more scheduled manner, is not well documented.

The aim of the present study was to study the factors associated with the acquisition of MRSA after LDLT in adults assessed by surveillance cultures obtained from multiple sites, including nares, and to analyze the risk factors by multivariate analysis.

\section{Methods \\ Patients}

We retrospectively reviewed the data from 171 patients that underwent LDLT at the University of Tokyo Hospital, a 1150-bed teaching hospital, between August 2001 and November 2004. Of 171 patients, 13 were colonized with MRSA preoperatively and were excluded from the study. The median patient age was 51 years (range, 19-67). The indications for LDLT in these patients included hepatitis $\mathrm{C}$ $(\mathrm{n}=53)$, hepatitis $B(\mathrm{n}=24)$, primary biliary cirrhosis ( $\mathrm{n}$ $=24)$, fulminant hepatitis $(n=18)$, biliary atresia $(n=8)$, autoimmune hepatitis $(\mathrm{n}=7)$, primary sclerosing cholangitis $(n=5)$, metabolic disease $(n=5)$, alcoholic cirrhosis $(n=4)$, cryptogenic cirrhosis $(n=2)$, and others $(n=8)$. Of the 158 patients, 68 had hepatocellular carcinoma. The median Child-Pugh score and model for end stage liver diseases (MELD) score of those patients was 10 (range, 514 ) and 13 (range, -3 to 48 ), respectively. The microbiologic and medical records of the patients from admission to 3 months after LDLT were reviewed. The present study was approved by The University of Tokyo Ethical Committee. The data used for the study are publicly available.

\section{Donor selection}

Donors were selected from the patients' relatives. Age, blood type, graft size, and liver function were also taken into consideration. $\mathrm{ABO}$ blood groups were required to be identical to or compatible with that of the recipients. The graft type was determined according to the ratio of the estimated graft volume to the recipient's standard liver volume ratio $[8,9]$. Our surgical technique for recipient and donor surgery is described elsewhere [10]. Donors were not routinely screened for Staphylococcus aureus perioperatively.

\section{Perioperative management}

Antimicrobial prophylaxis consisted of intravenous cefotaxime ( $1.0 \mathrm{~g}$ just before surgery, followed by $1.0 \mathrm{~g}$ every 6 hours intraoperatively and thereafter), ampicillin/sulbactam ( $1.0 \mathrm{~g}$ just before surgery, followed by $1.5 \mathrm{~g}$ every 12 hours intraoperatively and thereafter), and gentamicin, $60 \mathrm{mg}$ every 12 hours after surgery) for 5 days.

To prevent fungal infection, fluconazole (200 mg every 24 hours) was administered intravenously for 7 days after surgery. All patients received the same immunosuppressive regimens using tacrolimus (Prograf, Astellas Pharmaceutical Corporation, Tokyo, Japan) and methylprednisolone (Solu-Medrol, Pfizer Inc., New York, $\mathrm{NY}$ ). The details of the regimen are reported elsewhere [11].

\section{Definition of MRSA colonization}

All the patients were screened preoperatively for Staphylococcus aureus on admission for LDLT. Follow-up specimens were collected twice a week during the first month after LDLT, and thereafter once a week during the hospital stay. Routine surveillance specimens consisted of swabs of the anterior nares, pharynx, sputum, urine, and stool. In addition, swabs of wound or skin lesions, bile, and discharge from the abdominal cavity were collected postoperatively. Blood samples, collected percutaneously, and a segment of a removed intra-vascular catheter were also submitted when infection was suspected as the followings: fever $\left(>38^{\circ} \mathrm{C}\right)$, chills, or hypotension. Other clinical samples were added in patients with suspected infection according to the discretion of the attending physician.

Specimens were plated onto mannitol-salt agar or sheep blood agar. Staphylococcus aureus was identified using standard microbiologic methods.

Methicillin resistance was determined using a disk diffusion test performed on Mueller-Hinton agar after incubation for 24 to 48 hours at $30^{\circ} \mathrm{C}$. By the microdilution method, strains with an oxacillin minimum inhibitory concentration value of at least $4 \mu \mathrm{g} / \mathrm{ml}$ were defined as MRSA [12]. Patients colonized with Staphylococcus aureus at any site, and at any time during the hospital stay, were considered carriers.

\section{Definition of MRSA infection}

Nosocomial infections were defined according to the reports from the Centers for Disease Control and Prevention in 1988 and in 1992, as described elsewhere [13,14]. When MRSA was isolated from culture samples in the 
presence of nosocomial infection and other pathogenic organisms were absent, MRSA infection was diagnosed.

\section{Management of precaution for transmission of MRSA}

$0.2 \%$ benzalkonium chloride ethanol solution (Welpas, Maruishi Pharmaceutical Corporation, Oosaka, Japan) were used for hand hygiene of patients, medical, and nonmedical staffs in contact with patients. Contact precautions were taken in cases with MRSA colonization and/or infection. Eradication therapy such as intranasal mupirocin was not routinely performed. The screening of medical staffs for detection of MRSA was not also performed during the study period.

\section{Background and clinical data collection}

Background and clinical data collected for each patient included:

1) preoperative variables (age, gender, etiology of the underlying liver disease, presence of hepatocellular carcinoma, Child-Pugh score, MELD score, presence of ascites, use of diuretics, presence of encephalopathy, the international normalized ratio of prothrombin time level, serum bilirubin level [mg/dl], serum albumin level [g/dl], serum creatinine level [mg/dl], use of steroid, use of antimicrobials during the month before LDLT, presence of diabetes mellitus, history of hospital stay during the 6 months before LDLT, and methicillin-susceptible Staphylococcus aureus colonization;

2) surgical variables (operation time [hours], estimated blood loss [ml], blood transfusion [ml], graft volume/ standard liver volume ratio [\%], and application of duct to duct biliary reconstruction;

3) postoperative variables (length of urinary catheter insertion [days], length of arterial catheter insertion [days], length of central venous catheter insertion [days], length of endotracheal tube insertion [days], necessity for reoperation, acute rejection, cytomegalovirus infection, fungal infection, and postoperative use of antimicrobials other than the routine perioperative prophylaxis); and

4) pre- and postoperative variables (length of intensive care unit stay [days], and application of dialysis and/or apheresis).

\section{Statistical analysis}

Quantitative variables are presented as median and range. Categorical variables are presented as absolute counts. Univariate analysis was used to identify associations between each of the variables recorded and postoperative acquisition of MRSA. Wilcoxon rank sum test was used to compare the quantitative variables. Chi-square test or
Fisher's exact test was used to compare the categorical data.

For multivariate analysis, only variables with a $p$ value of less than 0.25 in the univariate analysis were entered into a logistic regression model by the backward-elimination procedure. The final regression model included covariates associated with a likelihood ratio of $p$ less than 0.15 . The results of the logistic regression were reported as odds ratios with $95 \%$ confidence intervals. A $p$ value of less than 0.05 was considered statistically significant. All statistical analyses were performed using the JMP5.1 software package (SAS Institute Inc., Cary, NC).

\section{Results}

\section{Acquisition of MRSA after LDLT}

The median number of screening samples for each patient and the compliances with surveillance culture for nares, pharynx, sputum, urine, and stool were 9 (range, 1-25), 9 (range, 0-25), 5 (range, 0-25), 9 (range, 1-24), and 6 (range, 0-22) samples, and 82\%, 82\%, 50\%, 80\%, and $60 \%$, respectively. Data on the detection of postoperative acquisition of MRSA are summarized in Figure 1. Postoperative acquisition of MRSA was detected in 35 of 158 patients $(22 \%)$ during the study period. The median period of time between LDLT and detection of MRSA was postoperative day 18 (range, 1-89 days). In 8 of 35 patients, MRSA was detected during the intensive care unit stay. Median length of hospital stay after LDLT were 45 (range, 6-90) days in patients without MRSA acquisition and 59 (range, 33-90) in those with MRSA acquisition, respectively $(p=0.0006)$. Eleven of $158(7 \%)$ patients developed MRSA infection during the study period: deep incisional surgical site infection (SSI) in 5, organ/space SSI in 2, intraabdominal infection in 2, lower respiratory infection in 1, and primary bloodstream infection in 1 patient, respectively. MRSA infections were eventually

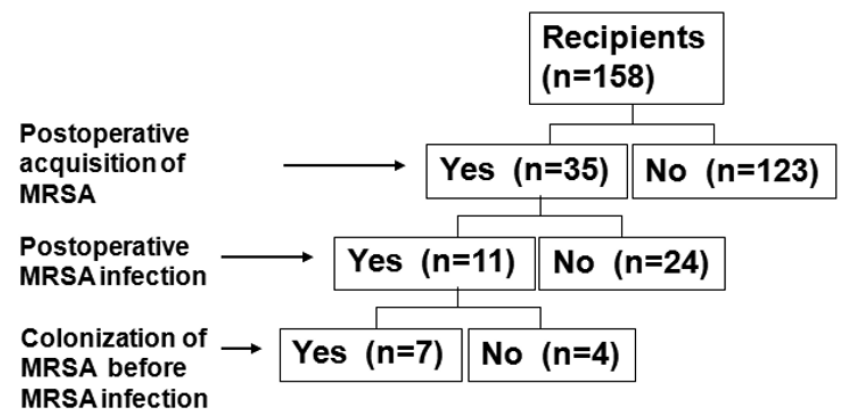

Figure I

The patient profile of postoperative MRSA colonization and infection Abbreviations: MRSA, methicillinresistant Staphylococcus aureus; LDLT, living donor liver transplantation. 
diagnosed in 7 of 31 patients (23\%) who were colonized with MRSA while only 4 of 127 subjects (3\%) with negative surveillance cultures developed MRSA infection ( $\mathrm{p}=$ $0.01)$. Table 1 shows the frequency of detection of MRSA from different clinical and surveillance specimens. In 30 subjects (86\%) MRSA was isolated in more than 2 sites.

\section{Risk factors for the Acquisition of MRSA after LDLT}

The results of the univariate analyses assessing the association between the acquisition of MRSA and clinical covariates are shown in an additional file 1. Age of at least 60 years $(p=0.01)$, presence of an endotracheal tube for at least 3 days $(p=0.03)$, and perioperative dialysis and/or apheresis $(p=0.008)$ were significant factors affecting the acquisition of MRSA. In the multivariate analyses (Table 2), 10 risk factors with a univariate $p$ value of less than 0.25 were entered into a logistic regression model by the backward-elimination procedure. In the final model, age of at least 60 years and perioperative dialysis and/or apheresis predicted the postoperative acquisition of MRSA. In contrast, postoperative use of fluoroquinolone was negatively associated with acquisition of MRSA.

\section{Discussion}

To date, this is the largest series study of the presence of MRSA after LDLT in adults. Of 158 patients, 35 (22\%) presented a positive culture for MRSA by median postoperative day 18 . The rate in the present study was higher than that in the recently published prospective study in DDLT [7], in which 9 of 60 (15\%) patients acquired nasal MRSA colonization by median postoperative day 24. Patients who acquired MRSA were significantly associated with an increased length of hospital stay in the present study. Similary, Singh et al. reported that an increased length of hospital stay was associated with new Staphylococcus aureus carriage acquisition in DDLT[15]. Longer hospital stay, which is one of a marker for greater severity of illness, also might have identified high risk candidates requiring more

Table I: Frequency of MRSA detection in different surveillance and clinical specimens of 35 patients

\begin{tabular}{ll}
\hline Sites & Number of patients (\%) \\
\hline Nares & $24(69 \%)$ \\
Pharynx & $21(60 \%)$ \\
Sputum & $18(51 \%)$ \\
Stool & $18(51 \%)$ \\
Urine & $11(31 \%)$ \\
Wound & $8(23 \%)$ \\
Intraabdominal drain & $5(14 \%)$ \\
Bile & $3(9 \%)$ \\
Intravascular catheter & $2(6 \%)$ \\
Ascites & $2(6 \%)$ \\
Pleural effusion & $2(6 \%)$ \\
Blood & $1(3 \%)$ \\
\hline
\end{tabular}

Thirty of 35 patients had MRSA detected from multiple sites. intensive care, which could lead an increasing chance of MRSA transmission. Although the anterior nares is the most frequent carriage site for Staphylococcus aureus [16], other extra-nasal sites such as skin, perineum, pharynx, gastrointestinal tract, vagina, and axillae can harbor the organism $[5,16]$. MRSA from the nares was detected in 24 of $158(15 \%)$ patients in the present study, which was comparable to the result of the previous report [7]. Of the 31 patients with MRSA-positive cultures, 7 (23\%) subsequently developed MRSA infection. In addition, 7 of 11 (64\%) patients who developed MRSA infection were colonized with MRSA prior to infection while only 4 of the 127 subjects (3\%) with negative surveillance cultures developed MRSA infection ( $p=0.001)$. This is in line with the findings of other authors, which indicated that interventions aimed at curtailing the transmission of MRSA may have a beneficial impact on the incidence of MRSA infection $[17,18]$. Furthermore, this is useful information in that it allows for earlier administration of a more appropriate antibiotic such as vancomycin in patients suspected of having MRSA infection.

The present study indicated that age of at least 60 years increased the risk of postoperative acquisition of MRSA by multivariate analysis. As a large number of variables $(\mathrm{N}=$ 39) were included in the analyses in the present study, we must recognize the possibility that statistical association might have occurred by chance. The exact reason why older patients acquire MRSA more frequently after LDLT is unclear. Some previous studies $[19,20]$ reported that older age was a risk factor for MRSA acquisition during hospitalization, although the interpretation was not described.

Another risk factor indicated by the present study, dialysis and/or apheresis, requires indwelling devices such as intravascular catheters. Invasive procedures are "entrance gates" for microorganisms, and potential hand contamination of personnel who perform these procedures might increase the risk of MRSA transmission [19]. On the other hand, perioperative dialysis and/or apheresis might merely be suggestive of the intensity of care required for patients in the present study. Perioperative dialysis and/or apheresis, mostly indicated in cases of deteriorated liver dysfunction in the present study, might suggest the deteriorated general conditions of patients, making them more prone to infectious diseases. Intensity of care required can be considered a surrogate marker for a number of manipulations that are major risk factors for MRSA transmission [21].

It might be better to adopt additional strategies for patients with these risk factors of MRSA acquisition. Singh et al. [15] reported an impact of an aggressive infection control strategy on Staphylococcus aureus infection in liver transplant recipients, including use of surveillance cul- 
Table 2: Multivariate analysis of risk factors for the acquisition of MRSA after LDLT

\begin{tabular}{lll}
\hline Variable & $\begin{array}{l}\text { Odds Ratio } \\
\text { (95\% Confidence interval) }\end{array}$ & $p$ Value \\
\hline Age $>=60$ & $3.33(1.17-9.58)$ & 0.03 \\
Duct to duct biliary reconstruction & $3.18(0.92-15.22)$ & 0.07 \\
Endotracheal tube (day) $>=3$ & $2.26(0.87-5.84)$ & 0.09 \\
Postoperative use of beta lactam & $0.49(0.20-1.23)$ & 0.13 \\
Postoperative use of fluroquinolone & $0.14(0.007-0.88)$ & 0.03 \\
Perioperative dialysis and/or apheresis & $2.92(1.16-7.39)$ & 0.02 \\
\hline
\end{tabular}

Abbreviations: MRSA, methicillin-resistant Staphylococcus aureus; LDLT, living donor liver transplantation.

tures to detect nasal and rectal colonization, use of cohort and contact isolation precautions, decolonization with intranasal mupirocin therapy, and educating patients and visitors about hand hygiene and MRSA transmission. In that study, the rate of new acquisition of Staphylococcus aureus decreased from $46 \%$ during the pre-intervention period to $10 \%$ during the post-intervention period, and the rate of Staphylococcus aureus infection decreased from $40 \%$ to $4 \%$, respectively.

The intensity of the use of antimicrobials, measured by the presence of preoperative antibiotic use during the month before LDLT did not correlate with the acquisition of MRSA after transplantation in the present study. Furthermore, postoperative use of fluoroquinolone was negatively associated with acquisition of MRSA, which was contrary to our expectations. It was difficult to analyze whether the postoperative frequency of use of antimicrobials increased a risk of MRSA acquisition in the present study. Only the antimicrobials used before the first date of detection of MRSA were included in the analysis, which caused the difference of observation period for exposure to antimicrobials between patients with and without acquisition of MRSA. The median period of time between LDLT and detection of MRSA was postoperative day 18, and 17 of 35 (49\%) patients acquired MRSA within 2 weeks after the operation. Although there is little doubt that widespread use of antimicrobials provides multidrugresistant strains of MRSA with a selective survival advantage [22], the relation between MRSA and antimicrobials seems more complex in the current series. Some studies [23-29] failed to show such an association by multivariate analysis. In other studies [30-33], exposure to specific antimicrobials, such as third generation cephalosporins, amoxicillin with clavulanic acid, quinolones, and other broad-spectrum antibiotics, increased the risk of MRSA infection or colonization. Crowcroft et al. [33] found no association between total antimicrobial use and MRSA colonization or infection and suggested that the problem was the inappropriate use of antimicrobials, not excessive use. This discrepancy is probably due to the fact that in the present study all the patients received long courses of mul- tiple antimicrobials resulting in broad coverage, as perioperative prophylaxis per protocol, and it is therefore difficult to detect the effect of a specific antimicrobial. It might also be better for reducing the acquisition of MRSA to shorten prophylactic use of antimicrobials to a maximum of 48 hours as used in other transplant centers $[7,34]$.

One limitation of the present study is that the MRSA carriage pattern was not analyzed. Longitudinal studies have distinguished three Staphylococcus aureus carriage patterns in healthy individuals $[15,35]$. This distinction is important because persistent carriers have higher Staphylococcus aureus loads and a higher risk of acquiring Staphylococcus aureus infection [36].

Another limitation of the present study is that we could not differentiate the specific MRSA strains. Pulsed-field gel electrophoresis analysis was not accessible and the data were not obtained. Therefore, we could not analyze the impact of MRSA transmission, such as patient-to-patient transmission by transient carriage on the hands of the medical staff in detail. Our observation remains speculative on this point. Similarly, it was impossible to know whether infection was due to the same strain as that of the colonization or to a newly acquired strain. Chang et al. [4] analyzed the isolates from infected sites and from the anterior nares in seven patients with MRSA infection, and reported that the same isolates were detected. Such detailed analyses might yield further information to prevent the spread of MRSA following LDLT.

\section{Conclusion}

There is a high incidence of MRSA early after LDLT in adults. Surveillance cultures should be performed periodically after LDLT to identify and prevent the transmission of MRSA.

\section{Abbreviations}

LDLT: living donor liver transplantation; DDLT: deceased donor liver transplantation; MELD: model for end stage liver diseases; MRSA: methicillin-resistant Staphylococcus 
aureus; MSSA: methicillin-susceptible Staphylococcus aureus.

\section{Competing interests}

The authors declare that they have no competing interests.

\section{Authors' contributions}

$\mathrm{MH}, \mathrm{YS}$, and MM designed the Research project and gave a critical view of manuscript writing. JK, YM, JT, KM, and KK helped in collecting the specimens, and the microbiologic and medical records. MH, YS, and ST wrote the manuscript. All the authors have read and approved the final manuscript.

\section{Additional material}

\section{Additional file 1}

An additional table. Association between postoperative acquisition of MRSA and perioperative variables by the univariate analysis.

Click here for file

[http://www.biomedcentral.com/content/supplementary/14712334-8-155-S1.doc]

\section{Acknowledgements}

This work was in part supported by a Grant-in-aid for Scientific Research from the Ministry of Education, Culture, Sports, Science, and Technology of Japan and Grants-in-aid for Research on HIVIAIDS, and Research on Measures for Intractable Diseases from the Ministry of Health, Labor and Welfare of Japan.

\section{References}

I. Singh N, Paterson DL, Chang FY, Gayowski T, Squier C, Wagener MM, Marino IR: Methicillin-resistant Staphylococcus aureus: The other emerging resistant gram-positive coccus among liver transplant recipients. Clin Infect Dis 2000, 30:322-327.

2. Singh N, Gayowski T, Rihs JD, Wagener MM, Marino IR: Evolving trends in multiple-antibiotic-resistant bacteria in liver transplant recipients: A longitudinal study of antimicrobial susceptibility patterns. Liver transpl 200I, 7:22-26.

3. Bert F, Galdbart JO, Zarrouk V, Le Mee J, Durand F, Mentre F, Belghiti J, Lambert-Zechovsky N, Fantin B: Association between nasal carriage of Staphylococcus aureus and infection in liver transplant recipients. Clin Infect Dis 2000, 3 I: I 295-I 299.

4. Chang FY, Singh N, Gayowski T, Drenning SD, Wagener MM, Marino IR: Staphylococcus aureus nasal colonization and association with infections in liver transplant recipients. Transplantation 1998, 65: I169-1172.

5. Desai D, Desai N, Nightingale P, Elliott T, Neuberger J: Carriage of methicillin-resistant Staphylococcus aureus is associated with an increased risk of infection after liver transplantation. Liver Transpl 2003, 9:754-759.

6. Davis KA, Stewart JJ, Crouch HK, Florez CE, Hospenthal DR: Methicillin-resistant Staphylococcus aureus (MRSA) nares colonization at hospital admission and its effect on subsequent MRSA infection. Clin Infect Dis 2004, 39:776-782.

7. Santoro-Lopes G, de Gouvea EF, Monteiro RC, Branco RC, Rocco JR, Halpern M, Ferreira AL, de Araujo EG, Basto ST, Silveria VG, RibeiroFilho J: Colonization with methicillin-resistant Staphylococcus aureus after liver transplantation. Liver Transpl 2005, I I:203-209.

8. Urata K, Kawasaki S, Matsunami H, Hashikura Y, Ikegami T, Ishizone $\mathrm{S}$, Momose $\mathrm{Y}$, Komiyama A, Makuuchi M: Calculation of child and adult standard liver volume for liver transplantation. Hepatology 1995, 2 I: I317-1321.

9. Kokudo N, Sugawara Y, Imamura H, Sano K, Makuuchi M: Tailoring the type of donor hepatectomy for adult living donor liver transplantation. Am J Transpl 2005, 5: I694-I703.

10. Sugawara $Y$, Makuuchi $M$ : Technical advancement in livingrelated liver transplantation. J Hepatobiliary Pancreat Surg 1999, 6:245-253.

I I. Sugawara Y, Makuuchi M, Kaneko J, Ohkubo T, Imamura H, Kawarasaki $\mathrm{H}$ : Correlation between optimal tacrolimus doses and the graft weight in living donor liver transplantation. Clin Transplant 2002, I 6:102-106.

12. Clinical and Laboratory Standards Institute: MI00-SI7. Performance standards for antimicrobial susceptibility testing; I7th informational supplement. Clinical and Laboratory Standards Institute. Wayne, PA 2007.

13. Garner JS, Jarvis WR, Emori TG, Horan TC, Hughes JM: CDC definitions for nosocomial infections. Am J Infect Control 1988, 16: I 28-140.

14. Horan TC, Gaynes RP, Martone WJ, Jarvis WR, Emori TG: CDC definitions of nosocomial surgical site infections, 1992: a modification of CDC definition of surgical wound infections. Infect Control Hosp Epidemiol 1992, 13:606-608.

15. Singh N, Squier C, Wannstedt C, Keyes L, Wagener MM, Cacciarelli TV: Impact of an aggressive infection control strategy on endemic Staphylococcus aureus infection in liver transplant recipients. Infect Control Hosp Epidemiol 2006, 27:122-126.

16. Williams RE: Healthy carriage of Staphylococcus aureus: its prevalence and importance. Bacteriol Rev 1963, 27:56-7I.

17. Muto CA, Jernigan JA, Ostrowsky BE, Richert HM, Jarvis WR, Boyce JM, Farr BM: SHEA Guideline for preventing nosocomial transmission of multidrug-resistant strains of Staphylococcus aureus and enterococcus. Infect Control Hosp Epidemiol 2003, 24:362-386.

18. Tomic V, Svetina Sorli P, Trinkaus D, Sorli J, Widmer A, Trampuz A: Comprehensive strategy to prevent nosocomial spread of methicillin-resistant Staphylococcus aureus in a highly endemic setting. Arch Intern Med 2004, I 64:2038-2043.

19. Asensio A, Guerrero A, Quereda C, Lizan M, Martinez-Ferrer M: Colonization and infection with methicillin-resistant Staphylococcus aureus: associated factors and eradication. Infect Control Hosp Epidemiol 1996, I 7:20-28.

20. Lucet JC, Paoletti X, Lolom I, Paugam-Burtz C, Trouillet JL, Timsit JF, Deblangy C, Andremont A, Regnier B: Successful long-term program for controlling methicillin-resistant Staphylococcus aureus in intensive care units. Intensive Care Med 2005, 3 1:1051-1057.

21. Dziekan G, Hahn A, Thune K, Schwarzer G, Schafer K, Daschner FD, Grundmann H: Methicillin-resistant Staphylococcus aureus in a teaching hospital: investigation of nosocomial transmission using a matched case-control study. J Hosp Infect 2000, 46:263-270.

22. Boyce JM: Understanding and controlling methicillin-resistant Staphylococcus aureus infections. Infect Control Hosp Epidemiol 2002, 23:485-487.

23. Crossley K, Landesman B, Zaske D: An outbreak of infections caused by strains of Staphylococcus aureus resistant to methicillin and aminoglycosides. II. Epidemiologic studies. J Infect Dis 1979, I39:280.

24. Peacock JE Jr, Marsik FJ, Wenzel RP: Methicillin-resistant Staphylococcus aureus: introduction and spread within a hospital. Ann Intern Med 1980, 93:526-532.

25. Hershow RC, Khayr WF, Smith NL: A comparison of clinical virulence of nosocomially acquired methicillin-resistant and methicillin-sensitive Staphylococcus aureus infections in a university hospital. Infect Control Hosp Epidemiol 1992, I 3:587-593.

26. French GL, Cheng AF, Ling JM, Mo P, Donnan S: Hong Kong strains of methicillin-resistant and methicillin-sensitive Staphylococcus aureus have similar virulence. J Hosp Infect 1990, I5: I 17-I 25.

27. Marshall C, Wolfe R, Kossmann T, Wesselingh S, Harrington G, Spelman D: Risk factors for acquisition of methicillin-resistant Staphylococcus aureus (MRSA) by trauma patients in the intensive care unit. J Hosp Infect 2004, 57:245-252.

28. Colleo R, Glynn JR, Gaspar C, Picazo JJ, Feres J: Risk factors for developing clinical infection with methicillin-resistant Sta- 
phylococcus aureus (MRSA) amongst hospital patients initially only colonized with MRSA. J Hosp Infect 1997, 37:39-46.

29. Topeli A, Unal S, Akalin HE: Risk factors influencing clinical outcome in Staphylococcus aureus bacteraemia in a Turkish University Hospital. Int J Antimicrob Agents 2000, 14:57-63.

30. Graffunder EM, Venezia RA: Risk factors associated with nosocomial methicillin-resistant Staphylococcus aureus (MRSA) infection including previous use of antimicrobials. J Antimicrob Chemother 2002, 49:999-1005.

31. Weber SG, Gold HS, Hooper DC, Karchmer AW, Carmeli Y: Fluoroquinolones and the risk for methicillin-resistant Staphylococcus aureus in hospitalized patients. Emerg Infect Dis 2003, 9:1415-1422.

32. Onorato M, Borucki MJ, Baillargeon G, Paar DP, Freeman DH, Cole CP, Mayhall CG: Risk factors for colonization or infection due to methicillin-resistant Staphylococcus aureus in HIV-positive patients: a retrospective case-control study. Infect Control Hosp Epidemiol 1999, 20:26-30.

33. Crowcroft NS, Ronveaux O, Monnet DL, Mertens R: Methicillinresistant Staphylococcus aureus and antimicrobial use in Belgian hospitals. Infect Control Hosp Epidemiol 1999, 20:3 I-36.

34. Singh N, Gayowski T, Wagener MM, Marino IR: Bloodstream infections in liver transplant recipients receiving tacrolimus. Clin Transpl 1997, I I:275-28I.

35. Kluytmans J, van Belkum A, Verbrugh H: Nasal carriage of Staphylococcus aureus: epidemiology, underlying mechanisms, and associated risks. Clin Microbiol Rev 1997, 10:505-520.

36. Nouwen JL, Fieren MW, Snijders S, Verbrugh HA, Belkum A: Persistent (not intermittent) nasal carriage of Staphylococcus aureus is the determinant of CPD-related infections. Kidney Int 2005, 67:1084-1092.

\section{Pre-publication history}

The pre-publication history for this paper can be accessed here:

http://www.biomedcentral.com/1471-2334/8/155/pre

pub

\section{Publish with Bio Med Central and every scientist can read your work free of charge}

"BioMed Central will be the most significant development for disseminating the results of biomedical research in our lifetime. "

Sir Paul Nurse, Cancer Research UK

Your research papers will be:

- available free of charge to the entire biomedical community

- peer reviewed and published immediately upon acceptance

- cited in PubMed and archived on PubMed Central

- yours - you keep the copyright 